\title{
Una montagna di debiti. L'Italia e la gestione del debito pubblico tra le due guerre
}

\author{
Marianna Astore*
}

1. Una panoramica dell'evoluzione del debito pubblico in Italia

La profonda recessione causata dalla pandemia di Covid-19 sta richiedendo misure eccezionali da parte dei governi e delle banche centrali di tutto il mondo. L'aumento significativo del debito pubblico, necessario in fase di emergenza per impedire il collasso delle strutture sanitarie e poi per assorbire la perdita di reddito a cui va incontro il settore privato, è stato inevitabile e livelli molto più alti di debito pubblico diventeranno una caratteristica permanente delle nostre economie ${ }^{1}$. Quella italiana non fa eccezione $\mathrm{e}$, anzi, il coronavirus peserà, $\mathrm{e}$ anche tanto, sulla finanza pubblica. Secondo le ultime stime, nel 2020 il debito pubblico supererà di 194 miliardi i livelli di fine 2019, con un peggioramento di 151,3 miliardi del saldo primario rispetto al 2019 (da $+1,8 \%$ del Pil a $-7,3 \%$ ), determinato da una caduta delle entrate tributarie e da un'impennata della spesa $(95,1$ miliardi in più al netto degli interessi; $+11,7 \%)$ per gli ammortizzatori sociali e le altre misure anticrisi ${ }^{2}$. Del resto, la storia dell'Italia unita è caratte-

Università Bocconi di Milano.

1 M. Draghi, We face a war against coronavirus and must mobilise accordingly, «Financial Times», 25 marzo 2020, disponibile online all'indirizzo: <https://www.ft.com/content/ c6d2de3a-6ec5-11ea-89df-41bea055720b> (05/20).

2 Il presente saggio è stato concluso il 15 novembre 2020. Per le stime sulla finanza pubblica italiana si veda M. Rogari, G. Trovati, Nel 2020 il debito pubblico supererà di 194 miliardi i livelli difine 2019, «Il Sole 24 ore», 6 ottobre 2020. L'articolo è basato sulle stime contenute

Marianna Astore, Bocconi University, Italy, marianna.astore@unibocconi.it, 0000-0002-5324-7539

FUP Best Practice in Scholarly Publishing (DOI 10.36253/fup_best_practice)

Marianna Astore, Una montagna di debiti. L'Italia e la gestione del debito pubblico tra le due guerre, pp. 191-213, @ 2020 Author(s), CC BY 4.0 International, DOI 10.36253/978-88-5518-202-7.10, in Piero Barucci, Piero Bini, Lucilla Conigliello (edited by), I mille volti del regime. Opposizione e consenso nella cultura giuridica, economica e politica italiana tra le due guerre, (c) 2020 Author(s), content CC BY 4.0 International, metadata CC0 1.0 Universal, published by Firenze University Press (www.fupress.com), ISSN 2704-5919 (online), ISBN 978-88-5518-202-7 (PDF), DOI 10.36253/978-88-5518-202-7 
rizzata per una sua parte significativa da alti livelli di debito pubblico, anche se, con l'eccezione del primo dopoguerra, non della portata di quelli della crisi da Covid-19. In questo preciso momento storico risulta, dunque, particolarmente interessante dare uno sguardo al passato per trovarvi analogie in termini di soluzioni o, semplicemente, per approfondire un aspetto poco studiato.

Guardando nel complesso all'evoluzione del debito pubblico in Italia è possibile individuare cinque fasi di accumulazione e quattro di riduzione (Fig. 1).

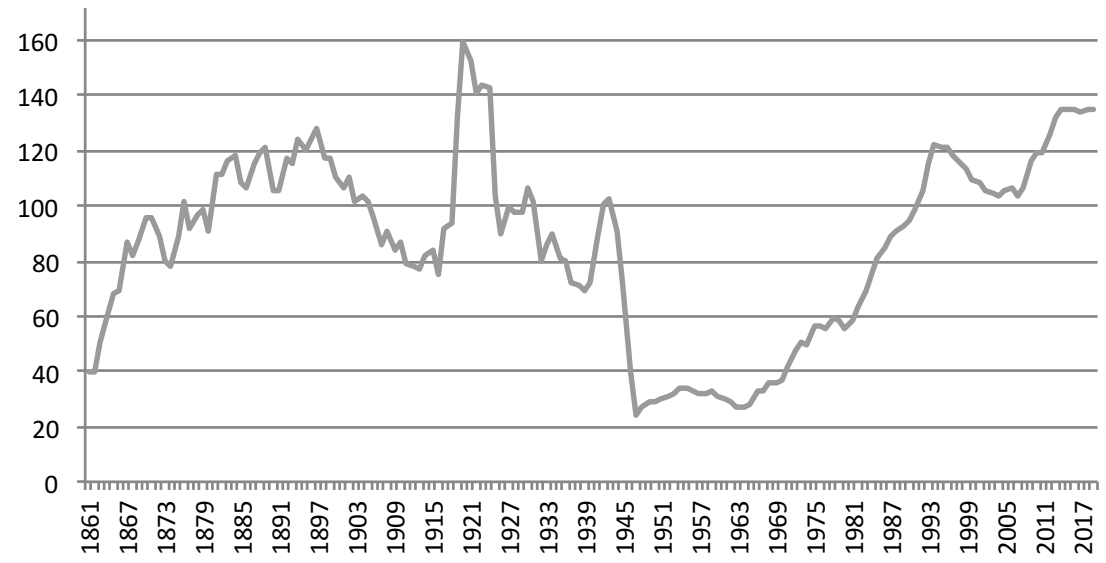

Figura 1 - Debito pubblico italiano in percentuale rispetto al Pil, 1861-2017. [Fonti: Per il debito pubblico 1861-2007: M. Francese, A. Pace, Il debito pubblico italiano dall'Unità a oggi. Una ricostruzione della serie storica, «Questioni di economia e finanza», Banca d'Italia, n. 31, 2008. Per il Pil 1861-2007: A. Baffigi, Italian national accounts: a project of Banca d'Italia, Istat, and University of Rome 'Tor Vergata', «Economic History Working Papers», Banca d'Italia, n. 18, 2011. Per i dati sul rapporto debito/Pil dal 2008 a oggi: Banca d'Italia, Base di dati statistica, disponibile online all'indirizzo: <https://infostat.bancaditalia.it/inquiry/home> (11/20)]

La prima fase di accumulazione del debito riguardò i primi trent'anni del nuovo Regno. Dal 1861 al 1876 fu causata - oltre che da parte dei debiti pregressi degli Stati preunitari, raccolti nel Gran libro del debito pubblico - da una vera e propria 'finanza di emergenza', dettata dalla costruzione, sia dal punto di vista materiale che istituzionale, dello Stato unitario. I politici della Destra storica tentarono di tenere il deficit di bilancio sotto controllo soprattutto dal punto di vista delle entrate e il pareggio di bilancio venne raggiunto sotto il dicastero di Quintino Sella, ma con la Sinistra storica, dal comportamento fiscale meno ortodosso rispetto alla Destra, ci fu una nuova crescita del debito dovuta prin-

nella nota di aggiornamento del DEF ed è disponibile online all'indirizzo <https://www. ilsole24ore.com/art/nel-2020-debito-pubblico-superera-194-miliardi-livelli-fine-2019-A$\mathrm{DOzDjt}>(11 / 20)$. 
cipalmente alle opere pubbliche romane, al risanamento di Napoli, alle costruzioni ferroviarie e all'abolizione del corso forzoso realizzata da Magliani (644 milioni di titoli speciali) ${ }^{3}$. Nel 1894 il debito pubblico raggiunse un 'picco' locale attestandosi al 126\% del Pil. Il rapporto scese agli inizi del XX secolo nel periodo giolittiano (prima fase di riduzione), grazie a una forte crescita del Pil ${ }^{4}$, e tornò a impennarsi durante la Prima guerra mondiale (seconda fase di accumulazione). Negli anni Venti l'effetto combinato di una politica di austerità, a cui seguì un grosso condono del debito estero da parte dei paesi alleati, fece scendere nuovamente il debito (seconda fase di riduzione), che però esplose nuovamente con la terza fase di accumulazione legata alla Seconda guerra mondiale. La disastrosa inflazione del secondo dopoguerra ridusse di circa 80 punti percentuali il rapporto debito/Pil (terza fase di riduzione). La quarta fase di accumulazione cominciò all'inizio degli anni Settanta del XX secolo e si protrasse fino al 1994, quando il rapporto debito/Pil arrivò a un nuovo massimo locale, poco sopra il $121 \%$. Le misure di bilancio prese sulla scorta delle regole fiscali del Trattato di Maastricht portarono a un processo di risanamento (quarta fase di riduzione), che però si è bruscamente interrotto a seguito della crisi finanziaria del 2008, prima, e dell'emergenza Covid, poi (quinta fase di accumulazione).

Se si guarda alle fasi di contenimento del debito, escludendo il boom giolittiano e le misure fiscali messe in atto per preparare l'entrata dell'Italia nell' $U$ nione monetaria europea, le due grandi contrazioni di debito/Pil ebbero luogo dal 1920 al 1939 e dal 1943 al 1947, ma per ragioni profondamente diverse (la crescita del Pil nel primo caso, l'inflazione nel secondo). Questo saggio si concentra sul primo episodio. Gli anni tra le due guerre mondiali offrono un laboratorio incredibilmente ricco per lo studio delle politiche monetarie e del debito pubblico. La Grande guerra segnò uno spartiacque, tra le altre cose, anche per il debito sovrano, con gli Stati Uniti da un lato, che emersero a livello internazionale come una delle principali nazioni creditrici, e i paesi europei sommersi dal debito, dall'altro. Il focus del lavoro è principalmente l'Italia, ma non sarà tralasciata la dimensione comparata del fenomeno, dando particolare attenzione alla componente estera del debito pubblico italiano.

\section{La politica di 'austerity destefaniana'}

La Prima guerra mondiale si accompagnò a un deciso aumento della spesa pubblica, comportando una sostanziale alterazione del Pil italiano. Nel complesso, i consumi crebbero del $20 \%$ come conseguenza del cospicuo aumento dei consumi

3 V. Zamagni, Il debito pubblico italiano 1861-1946: ricostruzione della serie storica, «Rivista di storia economica», 14 (3), 1998, pp. 207-210.

$4 \quad$ Il Pil reale crebbe in media dell'1,02\% tra il 1894 e il 1902 e del 2,43\% tra il 1903 e il 1913. Si vedano, anche per un confronto internazionale, i dati contenuti nel database di Jordà, Schularick e Taylor. Ò. Jordà, M. Schularick, A.M. Taylor, Macrofinancial History and the New Business Cycle Facts, in M. Eichenbaum e J.A. Parker (eds.), NBER Macroeconomics Annual 2016, Volume 31, University of Chicago Press, Chicago 2017. 
pubblici, che compensarono il calo di quelli privati, mentre investimenti ed esportazioni scesero di oltre il $50 \%{ }^{5}$. Il finanziamento del conflitto mondiale avvenne in misura prevalente con il ricorso al debito pubblico. Il rigore invocato da Einaudi per una maggior tassazione rimase inascoltato in quanto il sistema tributario italiano era tecnicamente insufficiente e, almeno inizialmente, tra i governanti era diffusa l'opinione, poi rivelatasi illusoria, che la guerra sarebbe stata di breve durata ${ }^{6}$. Come è possibile notare dalla tabella 1 , nel complesso del quinquennio considerato, l'indebitamento interno ed estero fornì allo Stato circa i due terzi delle nuove risorse finanziarie che gli erano necessarie; la tassazione e l'emissione monetaria coprirono all'incirca in egual misura il resto. Proporzioni simili sono riscontrabili per gli altri paesi belligeranti ${ }^{7}$. Al momento della firma del trattato di pace, lo stock del debito era pari a circa il 116 per cento del prodotto lordo del $1919^{8}$.

Tabella 1 - Incremento delle spese e delle risorse finanziarie dello Stato durante la guerra (milioni di lire, tra parentesi valori percentuali). [Fonte: G. Toniolo, La banca d'Italia e l'economia di guerra 1914-1919, in F. Cotula, M. De Cecco, G. Toniolo (a cura di), La banca d'Italia. Sintesi della ricerca storica, Laterza, Roma-Bari 2003, p. 174]

\begin{tabular}{llllll}
\hline \multirow{2}{*}{ Esercizio } & \multicolumn{4}{l}{ Incremento spese } & \multicolumn{3}{l}{ Incremento risorse finanziarie } \\
\cline { 2 - 6 } & $\begin{array}{l}\text { a prezzi } \\
\text { costanti }\end{array}$ & $\begin{array}{l}\text { a prezzi } \\
\text { correnti }\end{array}$ & $\begin{array}{l}\text { entrate } \\
\text { finali }\end{array}$ & $\begin{array}{l}\text { aumento } \\
\text { circolazione }\end{array}$ & $\begin{array}{l}\text { aumento } \\
\text { debito }\end{array}$ \\
\hline $1914-1915$ & 1716 & 2144 & -27 & 2103 & 68 \\
\hline \multirow{2}{*}{$1915-1916$} & 4502 & 7560 & 643 & 1330 & 5587 \\
\hline $1916-1917$ & 5136 & 13290 & 1643 & 1750 & 9897 \\
\hline $1917-1918$ & 5478 & 21830 & 3681 & 5134 & 130515 \\
\hline & & & $(16,9)$ & $(23,5)$ & $(59,6)$ \\
\hline $1918-1919$ & 6462 & 30883 & 6372 & 1607 & 22904 \\
\hline & & & $(20,6)$ & $(5,2)$ & $(74,2)$ \\
\hline TotALE & 23294 & 75707 & 12312 & 11924 & 51471 \\
\hline & & $(16,3)$ & $(15,8)$ & $(68,0)$ \\
\hline
\end{tabular}

5 A. Roselli, L'Italia e il finanziamento delle due guerre mondiali, in P. Barucci, P. Bini, L. Conigliello, Economia e Diritto in Italia durante il fascismo. Approfondimenti, biografie, nuovi percorsi di ricerca, Firenze University Press, Firenze 2017, p. 131.

6 Ivi, p. 134.

7 G. Toniolo, La banca d'Italia e l'economia di guerra 1914-1919, in F. Cotula, M. De Cecco, G. Toniolo (a cura di), La banca d'Italia. Sintesi della ricerca storica, Laterza, Roma-Bari 2003, pp. 174-175.

8 Ibidem. 
Per quanto riguarda l'accensione dei debiti, vanno in primo luogo citati i cinque 'prestiti nazionali' emessi tra il 1914 e il 1917. Il primo venne emanato alla fine del 1914, ancora in fase di neutralità, ma con l'intervento in guerra che era ormai considerato inevitabile; altri due prestiti furono emessi nel 1915 (ancora governo Salandra); il quarto nel 1917, mentre il quinto fu varato tra la fine del 1917 e l'inizio del 1918 (governo Orlando, con Nitti al Tesoro). I primi tre prestiti furono redimibili, gli altri due consolidati ${ }^{9}$. Un sesto prestito nazionale venne emesso nel 1919 , a guerra conclusa ${ }^{10}$. Allo stesso tempo, crebbero anche il debito a breve termine (con scadenza solitamente inferiore a due anni) e le anticipazioni staordinarie della Banca d'Italia ${ }^{11}$. I problemi più delicati riguardarono però la componente estera del debito. Come si può osservare dalla figura 2, immediatamente dopo la guerra il debito estero - costituito per la maggior parte da debiti di guerra nei confronti della Gran Bretagna e degli Stati Uniti - rappresentava più dell' $80 \%$ del Pil. L'esplosione della componente estera del debito non sorprende. In tempi normali, la grande maggioranza del debito italiano era collocato all'interno; al debito estero si ricorreva sempre in occasioni eccezionali, come negli anni immediatamente successivi all'unificazione, per ritornare al gold standard nei primi anni 1880, durante la crisi finanziaria dei primi anni $1890^{12}$. Dopo l'entrata in guerra dell'Italia, il primo accordo finanziario con la Gran Bretagna riguardò l'apertura di un credito di 50 milioni di sterline presso la Banca d'Inghilterra, che venne rinnovato più volte $^{13}$; con la partecipazione degli Stati Uniti al conflitto, l'Italia si rivolse principalmente a questi ultimi. I prestiti vennero utilizzati in larga parte per il pagamento delle importazioni di guerra italiane, ma anche per consentire interventi sui mercati valutari per mantenere stabile il tasso di cambio della lira. Finanziariamente la Prima guerra mondiale fu caratterizzata da una complessa ragnatela di prestiti tra le potenze alleate. Alla fine del conflitto, nel novembre 1918, l'indebitamento to-

9 Roselli, L'Italia e il finanziamento delle due guerre mondiali, cit., p. 138.

10 Più precisamente il primo prestito venne autorizzato il 19 dicembre 1914, era redimibile con rimborsi a partire dal 1925 e venne collocato a 97 lire con tasso d'interesse netto 4,5\%; il secondo fu autorizzato il 15 giugno 1915, con modalità analoghe al primo. Il terzo prestito nazionale, sempre redimibile, fu emesso il 22 dicembre 1915 al tasso d'interesse del 5\% e al prezzo di lire $97,50 \%$, mentre il quarto e il quinto prestito, emessi rispettivamente il 2 gennaio 1917 e il 6 dicembre del medesimo anno, erano dei consolidati 5\%. Un sesto prestito nazionale fu poi emesso il 24 novembre 1919, sempre in consolidato 5\% al prezzo di 87,50 lire, ma dei 21 miliardi circa raccolti, solo 7 furono pagati in contanti; l'operazione si configurò quindi più come un consolidamento parziale di altri debiti che non come un nuovo rastrellamento di fondi. Cfr. Ministero del tesoro, Direzione generale del debito pubblico, Relazione del direttore generale alla commissione parlamentare di vigilanza. Il debito pubblico in Italia, 1861-1987, vol. I, Istituto poligrafico e zecca dello Stato, Roma 1988, p. 31.

11 M. Marinkov, Conquering the Debt Mountain: Financial Repression and Italian Debt in the Interwar Period, in E. Dabla-Norris (ed.), Debt and Entanglements between the Wars, International Monetary Fund, Washington 2019, p. 181.

12 Zamagni, Il debito pubblico italiano 1861-1946, cit., p. 217.

13 G. Falco, L'Italia e la politica finanziaria degli alleati, 1914-1920, Ets, Pisa 1983, p. 57. 
tale ammontava a circa 21,6 miliardi di dollari ${ }^{14}$. Dal 1917 al 1922 i prestiti degli Stati Uniti agli Alleati ammontarono a 9387 milioni di dollari, di cui 4137 milioni alla Gran Bretagna, 2933 alla Francia e 1648 all' Italia; nel complesso questi tre paesi ricevettero il $93 \%$ dei prestiti statunitensi ${ }^{15}$. Terminato il conflitto, venne però subito manifestata la volontà statunitense di interrompere i prestiti ${ }^{16}$. Nel caso italiano alla decisione non furono estranei elementi geopolitici, in quanto si temeva che i crediti venissero utilizzati per dilazionare la smobilitazione nel tentativo di sostenere le pretese espansionistiche italiane nell'Adriatico ${ }^{17}$. Il mancato rinnovo dell'assistenza finanziaria anglo-americana fu un evento traumatico per l'Italia, che si trovò da sola ad affrontare i profondi squilibri economici causati dal conflitto.

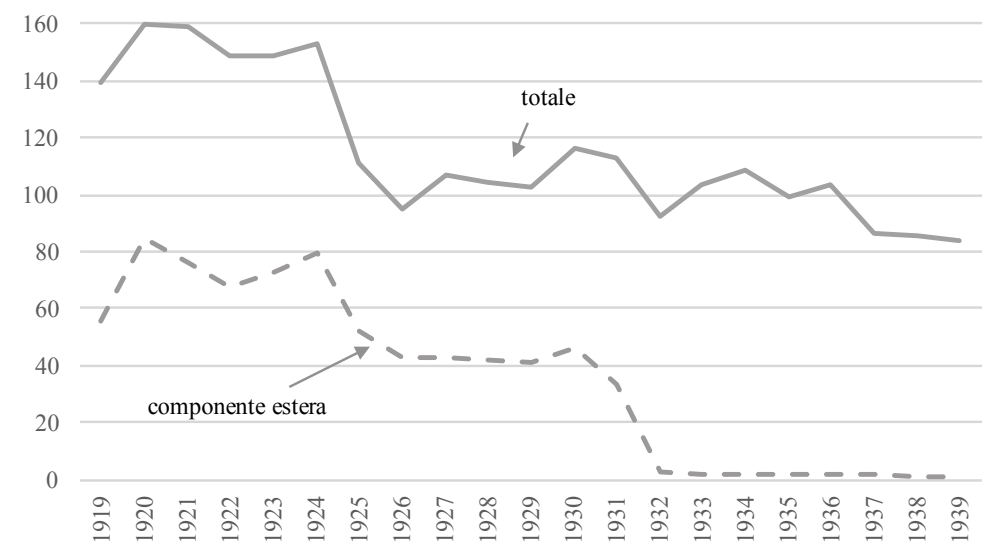

Figura 2 - Debito estero e totale del debito pubblico come percentuale del Pil, 19191939. [Fonte: M. Astore, M. Fratianni, 'We Can't Pay': How Italy Dealt with War Debts after World War I, «Financial History Review», 26 (2), 2019, p. 211]

14 Per una completa descrizione quantitativa dei crediti e debiti interalleati, si veda A. Roselli, Money and Trade Wars in Interwar Europe, Palgrave Macmillan, Basingstoke and New York 2014, p. 3.

15 B. Eichengreen, Till Debt Do Us Part: the U. S. Capital Market and Foreign Lending, 19201955, Working Paper n. 2394, National Bureau of Economic Research, Cambridge (MA), 1987 , tavola 5.

16 Così ricordava il direttore della Banca d'Italia Bonaldo Stringher: «Ero a Parigi e a Londra quando si concluse l'armistizio degli Alleati con la Germania, cioè nelle prime settimane di novembre 1918, e si è sentito subito dire, segnatamente e vibratamente dal Rappresentante del Tesoro federale degli Stati Uniti [Oscar T. Crosby], che chiusa la guerra, dovevano chiudersi necessariamente i crediti di Stato così per il nostro come per gli altri paesi debitori» (Promemoria di Bonaldo Stringher a Carlo Schanzer, fine febbraio 1920. Il testo del promemoria è riportato in Toniolo, La banca d'Italia e l'economia di guerra 1914-1919, cit., pp. 320-321).

17 A tal proposito Keynes scrisse: «Dal punto di vista americano la posizione è probabilmente commista con l'alta politica. Penso che il Tesoro degli Stati Uniti fornirebbero maggiore assistenza se le pretese territoriali italiane fossero moderate», cit. in D.J. Forsyth, La crisi dell'Italia liberale, Corbaccio, Milano 1998, p. 241. 
Nel complesso la struttura dell' indebitamento subì una profonda trasformazione: i debiti a medio e lungo termine (consolidati e redimibili) passarono dal $94,1 \%$ al $60,9 \%$ del totale, mentre il debito fluttuante perse la sua tradizionale funzione di strumento per far fronte a temporanee esigenze di cassa e la sua percentuale aumentò dal $5,9 \%$ al $39,1 \%{ }^{18}$. Negli anni post-bellici gli ultimi governi dell'Italia liberale che si susseguirono a ritmo ravvicinato dovettero affrontare, dunque, sul fronte della finanza pubblica due seri problemi contemporaneamente: l'eliminazione dei deficit e l'allungamento della scadenza del debito. La necessità di rimettere $\mathrm{i}$ conti in ordine era, del resto, un'esigenza non solo italiana, ma comune anche agli altri paesi belligeranti. Alla conferenza internazionale di Bruxelles, convocata nell'autunno 1920 con lo scopo di formulare programmi di stabilizzazione delle economie dei paesi usciti dalla guerra, le situazioni deficitarie di finanza pubblica in cui versavano quasi tutti i paesi europei furono rappresentate con allarme dai partecipanti e fu espressa l'esigenza di riportare i bilanci pubblici in pareggio soprattutto attraverso la diminuzione delle spese ${ }^{19}$.

Con l'avvento del primo governo fascista, il Ministro del tesoro e delle finanze Alberto de' Stefani, che mantenne il dicastero dal dicembre 1922 fino al luglio del $1925^{20}$, si trovò di fronte a questa pesante eredità di finanza pubblica. La politica destefaniana si articolò intorno a tre punti principali: il contenimento della dinamica salariale e l'accorta riapertura dei canali di credito; la ripresa di un liberismo doganale controllato e il raggiungimento del pareggio di bilan$\mathrm{cio}^{21}$. Il ministro non solo interpretò in maniera rigorosa le direttive di Mussolini, ma sfoggiò anche una notevole dose di pragmatismo nell'adattare le proprie idee di carattere teorico, sulla scia degli insegnamenti del suo maestro Maffeo Pantaleoni, con il contesto politico e sociale in cui era chiamato ad operare ${ }^{22}$. I tagli necessari al bilancio statale avvennero principalmente attraverso la privatizzazione del servizio telefonico e la riduzione dell'occupazione nell'ambito delle ferrovie, oltre che con una ristrutturazione della pubblica amministrazio-

18 D. Fausto, Lineamenti dell'evoluzione del debito pubblico in Italia (1861-1961), «Rivista di storia finanziaria $\gg, 15,2005$, p. 89.

19 P. Bini, Austerità e crescita negli anni 1922-1925 del fascismo. Alberto De' Stefani e l'ultima controffensiva del liberismo prima della resa all'economia corporativa, in P. Barucci, P. Bini, L. Conigliello (a cura di), Economia e Diritto in Italia durante il fascismo. Approfondimenti, biografie, nuovi percorsi di ricerca, Firenze University Press, Firenze 2017, p. 40.

20 Originariamente De' Stefani venne chiamato a dirigere il Ministero delle finanze, a cui si aggiunse il Tesoro, una volta morto nel dicembre dello stesso anno il suo originario titolare, il popolare Vincenzo Tangorra. F. Marcoaldi, De' Stefani Alberto, in Dizionario biografico degli italiani, vol. XXXIX, Istituto della Enciclopedia italiana, Roma 1991, ad nomen.

21 Sulla politica economica di De' Stefani si veda D. Fausto, La finanza pubblica fascista, in Id. (a cura di), Intervento pubblico e politica economica fascista, Franco Angeli, Milano pp. 579 585; Id., Lineamenti dell'evoluzione del debito pubblico in Italia (1861-1961), cit.; Marcoaldi, De' Stefani Alberto, cit.

22 L. Segreto, Giuseppe Volpi di Misurata al Ministero delle finanze: tecnocrate o politico?, in P. Barucci, P. Bini, L. Conigliello, Intellettuali e uomini di regime nell'Italia fascista, Firenze University Press, Firenze 2019, p. 15. 
ne, un'operazione molto complessa che fece ridurre a quasi un terzo il rapporto tra spesa pubblica e Pil (dal 36 al 13 per cento), oltre a generare una riduzione del rapporto tra debito e $\mathrm{Pil}^{23}$. L'avanzo di bilancio venne raggiunto nell'esercizio 1924-1925 grazie alla diminuzione delle spese per consumi pubblici e all'andamento dell'entrate, caratterizzate prima da una sostanziale stabilità e poi da una loro lieve crescita ${ }^{24}$. Il fascismo non esitò ad esaltare a fini propagandistici il raggiunto pareggio, ma al risultato non erano certamente estranei l'esaurirsi delle spese di guerra, la politica finanziaria dei governi precedenti, nonché alcuni astuti espedienti di bilancio. Va inoltre rilevato come la politica economica di quegli anni trasse beneficio dal ciclo positivo dell'economia internazionale e dalla relativa stabilità del tasso di cambio nominale della lira. Difatti, l'acuta turbolenza del dopoguerra, che aveva sottoposto la lira a violente fluttuazioni dal 1919 al 1923, sembrava essersi calmata e il cambio nominale rimase sostanzialmente stabile dalla metà del 1923 fino all'estate del 1924, mentre il tasso di cambio reale rispetto alle principali valute estere gradualmente si indeboli ${ }^{25}$. Il risultato fu che la diminuzione della spesa pubblica destefaniana, da cui sarebbero potuti scaturire effetti negativi a cascata, fu più che compensata dal forte incremento sia della domanda di investimenti che della domanda estera.

L'azione di De' Stefani andò incontro però anche a qualche insuccesso, specialmente per quanto riguarda il consolidamento del debito fluttuante. Nel febbraio 1924 venne emesso un prestito redimibile al 4,75\%, venticinquennale, che doveva servire al rimborso di Bot con l'obiettivo di sostituirli completamente. La prima emissione stabilita era di 5 miliardi di lire, ma si riuscì a raccogliere solo un miliardo e mezzo di capitale nominale. L'insuccesso dell'operazione ebbe ripercussioni sulla situazione della Tesoreria, perché, nonostante un aumento dei tassi sui buoni ordinari del Tesoro, ci fu una forte spinta a chiederne il rimborso. Fu un duro colpo per la politica di De' Stefani, che cercò di riparare con l'istituzione, il 26 dicembre 1924, dei buoni postali fruttiferi, una misura che darà risultati soltanto in seguito ${ }^{26}$. A ciò si aggiunsero le difficoltà monetarie. Mentre De' Stefani si compiaceva della tranquillità della lira nel marzo $1924^{27}$, agli osservatori più attenti, come Gustavo Del Vecchio, non era sfuggito che l'economia italiana si trovasse in una fase di espansione e fossero necessarie misure

23 Ibidem.

24 È necessario tuttavia sottolineare come De' Stefani non intendesse limitarsi a realizzare genericamente il pareggio di bilancio, volendone piuttosto conseguire una specifica modalità, ovvero quella basata sul taglio della spesa pubblica invece che sull'aumento delle imposte e tasse (Bini, Austerità e crescita negli anni 1922-1925 del fascismo, cit., p. 34).

25 M. De Cecco (a cura di), L'Italia e il sistema finanziario internazionale, 1919-1936, Laterza, Roma-Bari 1993, p. 48.

26 Ministero del tesoro, Direzione generale del debito pubblico, Relazione del direttore generale alla commissione parlamentare di vigilanza. Il debito pubblico in Italia, 1861-1987, vol. I, cit., p. 36.

27 F. Cotula, L. Spaventa (a cura di), La politica monetaria tra le due guerre, 1919-1935, Laterza, Roma-Bari 1993, p. 119. 
di restrizione monetaria ${ }^{28}$. La graduale scivolata del cambio iniziata nella seconda metà del 1924 si trasformò in una brusca caduta nella primavera del 1925, con la lira preda di una fortissima speculazione internazionale ${ }^{29}$. Così quando al deficit della bilancia dei pagamenti (aggravato dal pessimo raccolto del 1924 e dalle riduzioni delle rimesse degli emigrati) si aggiunse un rialzo dei valori azionari assolutamente spropositato, come reazione venne attuato un brusco aumento del tasso di sconto che portò a un crollo del corso dei titoli e a un allontanamento di De' Stefani, su esplicito invito della Confindustria. Gli ambienti industriali fecero sentire le loro preoccupazioni in un incontro con Mussolini avvenuto il 3 luglio. Qualche giorno più tardi, il 13 luglio, dopo che Mussolini tentò fino all'ultimo di convincere Alberto Pirelli ad accettare il ministero, De' Stefani fu sostituito da Giuseppe Volpi, una decisione che venne salutata con entusiasmo negli ambienti economici e da una ripresa in borsa inizialmente superiore alle aspettative ${ }^{30}$.

\section{Volpi e la sistemazione dei debiti di guerra}

Il neoministro Giuseppe Volpi ereditò dal suo predecessore l'irrisolto problema dei debiti di guerra, insieme a una situazione economica e politica alquanto complessa. Al momento dell'assunzione del dicastero, Volpi aveva già alle spalle una carriera molto lunga nel mondo degli affari oltre che come grand commis de l'État ${ }^{31}$. Dunque, se Volpi da un lato non aveva le competenze tecniche di De' Stefani, aveva certamente dalla sua molta esperienza nei negoziati di carattere politico-diplomatico. I debiti di guerra erano stati uno dei temi 'caldi' alla fine del conflitto, sia a livello di opinione pubblica che di dibattito governativo e numerose conferenze internazionali erano state organizzate per cercare di risolvere la vexata quaestio ${ }^{32}$. A metà degli anni Venti, l'accordo con Washington urgeva per una ripresa degli investimenti stranieri in Italia, in un momento in cui i mercati finanziari statunitensi si trovavano in una condizione di surplus di capitali ${ }^{33}$. Da questo punto di vista, i paesi europei erano i destinatari ideali, ma la mancata sistemazione del debito frenava non poco le attività delle banche americane in Europa, in particolare della Banca Morgan. Da parte italiana, un accordo Treves, Milano-Roma 1932 (1924), pp. 331-348.

29

30

31 Aveva fatto parte dell delegazione italiana che prese parte ai lavori per la sistemazione dell'economia internazionale a seguito del Trattato di Versailles e mantenuto per due anni la carica di vicepresidente del Comitato finanziario della Commissione economica suprema. Ivi, pp. 13-14.

32 M. Astore, M. Fratianni, 'We Can't Pay': How Italy Dealt with War Debts after World War I, «Financial History Review», 26 (2), 2019, pp. 197-222.

33 M. Fratianni, F. Giri, The Tale of two Great Crises, «Journal of Economic Dynamics and Control», 81, 2017, pp. 5-31. 
su tale questione era imprescindibile per potere anche solo ipotizzare qualsiasi progetto di carattere finanziario che avesse come interlocutori i banchieri americani, lungamente corteggiati nei primi anni Venti soprattutto dall'industria elettrica domestica, alla costante ricerca di capitali per potenziare gli impianti ${ }^{34}$.

I colloqui italo-statunitensi iniziarono il 18 giugno, in un momento di particolare debolezza della lira sui mercati valutari internazionali. Delegati italiani erano l'ambasciatore a Washington Giacomo De Martino e il direttore del credito italiano Mario Alberti, mentre il delegato americano era il segretario al Tesoro Andrew Mellon, seppur un importante ruolo nelle trattative fu rivestito dal banchiere Thomas William Lamont, rappresentante della casa Morgan ${ }^{35}$. Il primo progetto di sistemazione del debito italiano presentato da Alberti alla World War Foreign Debt Commission si basava sulla 'capacità di pagamento' dell'Italia e faceva esplicito riferimento all'accordo sul debito di guerra raggiunto tra la Gran Bretagna e gli Stati Uniti nel 1923. L'Italia - argomentava la delegazione - aveva una ricchezza media per abitante pari a neppure un terzo della ricchezza media per abitante inglese (586 dollari versus 2000 , non tenendo conto del dominio coloniale britannico) e su tale ridotta capacità di pagamento avrebbe dovuto basarsi l'accordo ${ }^{36}$. Tratto caratteristico del cosiddetto 'piano Alberti' era l'assoluta genericità in termini di impegni concreti di pagamento da parte dell'Italia, ma la proposta era stata presentata in maniera tale da non irritare l'opinione pubblica americana, assai sensibile al tema dei debiti di guerra: si dichiarava la disponibilità italiana a pagare capitale ed interessi, ma subordinando l'inizio dei pagamenti ad una serie di condizioni legate all'andamento dell'economia italiana e, in particolare, alla bilancia dei pagamenti e al tasso di cambio della lira. La delegazione italiana provò a contrattare in prima istanza una moratoria di dieci anni per il pagamento della prima rata o, quantomeno, una dilazione fino a quando il tasso di cambio della lira avesse raggiunto una quotazione di 20 lire per dollaro ${ }^{37}$. La proposta, ovviamente, era per molti versi inaccettabile dagli americani e le trattative entrarono in una situazione di stallo, di cui però non venne data comunicazione all'opinione pubblica. Quello che si richiedeva, in particolare, era la presentazione da parte della delegazione italiana di una documentazione statistica in grado di provare l'effettiva capacità di pagamento.

34 Segreto, Giuseppe Volpi di Misurata al Ministero delle Finanze: tecnocrate o politico?, cit., p. 17.

35 S. Romano, Giuseppe Volpi. Industria e finanza tra Giolitti e Mussolini, Marsilio, Venezia 1997, p. 133.

36 Archivio centrale dello Stato (d'ora in avanti Acs), Fondo Volpi, busta 10, fascicolo 85, Memoria del rappresentante italiano Mario Alberti presentata alla World War Foreign Debt Commission degli Stati Uniti d'America, giugno 1925. Il documento è riportato anche in De Cecco, L'Italia e il sistema finanziario internazionale 1919-1936, cit., pp. 560-564.

37 Nel giugno del 1925 il cambio si attestava invece su un livello pari a 26,106. Banca d'Italia, Portale dei tassi di cambio, consultabile online all'indirizzo $<$ https://tassidicambio.bancaditalia.it/> (11/20). 
Nel momento in cui Volpi assunse la guida del dicastero, nel luglio del 1925, il negoziato con gli Stati Uniti era dunque già cominciato ${ }^{38}$. Il neoministro decise però di non partire immediatamente per gli Stati Uniti: nell'estate del 1925 la svalutazione della lira era divenuta fonte di forte preoccupazione e, inoltre, occorreva preparare il dossier concernente la situazione economica italiana ${ }^{39}$. La missione ufficiale partì il 22 ottobre con una delegazione di dieci persone; il ministro era accompagnato, tra gli altri, da Dino Grandi, all'epoca sottosegretario di Stato per gli Affari esteri, dall'ambasciatore De Martino, da Alberto Pirelli e Mario Alberti ${ }^{40}$. Pirelli aveva già seguito la vicenda per conto del governo in maniera discreta, avendo partecipato in precedenza alla Conferenza di Londra del 1923, durante la quale erano state poste le prime basi per il negoziato sul debito. Per tale motivo Volpi insistette a lungo perché facesse parte della delegazione che doveva partire per gli Stati Uniti a fine ottobre e i due lavorarono fianco a fianco nei negoziati di Washington, trovando sempre un'intesa su tutto $^{41}$. Completavano la delegazione esperti attuariali e finanziari, tra cui il famoso statistico Corrado $\mathrm{Gini}^{42}$. Gini rivestì un'importanza fondamentale: assieme ai più importanti statistici italiani del tempo, come Giorgio Mortara, preparò una documentazione di circa seicento pagine, che descriveva nel dettaglio le condizioni demografiche, economiche e finanziarie dell'Italia ${ }^{43}$. Le argomentazioni facevano leva soprattutto sulle perdite subite dall'Italia in guerra e sulla bassa percentuale che le era stata attribuita sulle riparazioni (10\% contro il $52 \%$ alla Francia e il $22 \%$ alla Gran Bretagna). Veniva inoltre evidenziato come la bilancia commerciale italiana fosse sempre stata strutturalmente in passivo e la popolazione in aumento. La documentazione statistica non venne contestata e giocò un ruolo fondamentale nel raggiungimento dell'accordo, che venne siglato ufficialmente il 14 novembre 1925. Esso stabilì che il debito italiano venisse ridotto da 2148 milioni di dollari a 2042 milioni, con rimborsi su un arco temporale di 62 anni (contro i 90 richiesti inizialmente dall'Italia) e con tassi di interesse molto favorevoli che andavano dallo $0 \%$ per i primi cinque anni per poi salire gradualmente fino al $2 \%$ degli ultimi sette anni ${ }^{44}$. Il valore attuale dei rimborsi,

38 Romano, Giuseppe Volpi. Industria e finanza tra Giolitti e Mussolini, cit., p. 132.

39 Ibidem.

40 Acs, Fondo Volpi, busta 10, fascicolo 85, Mussolini al ministro delle finanze Volpi, 2 ottobre 1925.

${ }^{41}$ Segreto, Giuseppe Volpi di Misurata al Ministero delle Finanze: tecnocrate o politico?, cit., pp. 23-24.

42 Acs, Fondo Volpi, busta 10, fascicolo 85, Il ministro delle finanze Volpi a Mussolini, 9 ottobre 1925 .

43 J.G. Prévost e J.P. Beaud, Statistical expertise and the twilight of liberal in Italy, in J.G. Prévost and J.P. Beaud (eds.), Statistics, Public Debate and The State, 1800-1945. A Social, Political and intellectual History of Numbers, Pickering \& Chatto, London 2012, pp. 133-152.

44 G. Volpi di Misurata, Finanza fascista, Libreria del Littorio, Roma 1929, pp. 42-44; De Cecco, L'Italia e il sistema finanziario internazionale, 1919-1936, cit., documento 87, pp. 613-614. 
al tasso di sconto del 5\%, ammontava a 260 milioni di dollari. Gli inglesi, avendo una maggiore capacità di pagamento, non erano riusciti ad ottenere condizioni altrettanto favorevoli.

Concluso l'accordo con gli Stati Uniti, bisognava procedere con la sistemazione del debito inglese. La Gran Bretagna si trovava in una posizione finanziariamente più debole rispetto agli Stati Uniti. Solo l'anno precedente la sterlina era rientrata in regime di gold standard alla stessa parità dell'anteguerra, con una forte svolta deflativa per l'economia inglese, e il cancelliere dello Scacchiere Winston Churchill si oppose alla richiesta italiana che l'accordo dovesse avvenire alle stesse condizioni di quello statunitense ${ }^{45}$. L'accordo venne siglato il 27 gennaio 1926 e la cifra concordata fu di 276,75 milioni di sterline (valore non scontato), mentre il valore scontato dei pagamenti ammontava a 84 milioni di sterline; le rate, anche questa volta, vennero dilazionate nell'arco di 62 anni, ma, per andare incontro alle richieste inglesi, rimasero sostanzialmente costanti diversamente dall'agreement americano che prevedeva incrementi nel tempo ${ }^{46}$. Per l'Italia, tuttavia, il risultato finale fu peggiore di quello ipotizzato all'inizio di luglio del 1925, quando il Tesoro inglese si era detto pronto a concessioni maggiori; paradossalmente, alla luce del continuo miglioramento della situazione economico-finanziaria italiana, quei termini vennero rivisti al ribasso ${ }^{47}$. Il negoziato venne ulteriormente complicato dalla questione dell'oro italiano a Londra, versato durante la Prima guerra mondiale come garanzia per i prestiti britannici $^{48}$. Gli accordi finanziari con la Gran Bretagna del 1915 (il primo venne raggiunto a Nizza a giugno e il secondo a Londra a novembre) prevedevano l'apertura di un credito di 182 milioni di sterline, a fronte del quale il governo italiano si impegnò ad emettere buoni del Tesoro e a cedere 31,4 tonnellate d'oro (equivalenti a 22,2 milioni di sterline), che vennero depositate presso la Banca d'Inghilterra. Dopo la guerra, oltre ai debiti, bisognava definire la restituzione dell'oro. I primi negoziati si svolsero nel 1920-1921 e prevedevano che l'oro sarebbe stato trattenuto dalla Banca d'Inghilterra, fino a quando l'Italia non avesse ripagato i propri debiti. Con l'accordo di ristrutturazione del 1926, si stabilì la restituzione all'Italia, a partire dal 1928, dei suoi depositi in oro a Londra in proporzione ai pagamenti fatti per il rimborso dei debiti di guerra. La questione dell'oro italiano a Londra era particolarmente rilevante per il ritorno della lira alla convertibilità aurea che, come è noto, implicava un'emissione monetaria proporzionale alle riserve in metallo prezioso. Se l'oro italiano fosse stato considerato come un deposito presso la Banca d'Inghilterra, l'Italia avrebbe potuto includerlo contabilmente nelle proprie riserve ufficiali; se, al contrario, l'oro fosse stato considerato ceduto tramite un contratto di 'pronti contro termine'

45 Roselli, Money and Trade Wars in Interwar Europe, cit., p. 61.

46 Astore, Fratianni, 'We Can't Pay': How Italy Dealt with War Debts after World War I, cit., p. 205.

47 Segreto, Giuseppe Volpi di Misurata al Ministero delle finanze: tecnocrate o politico?, cit., p. 26.

48 Sulla questione dell'oro italiano a Londra si veda Roselli, Money and Trade Wars in Interwar Europe, cit., pp. 62-63. 
(sostanzialmente un repo, repurchase agreement), sarebbe appartenuto alla Banca d'Inghilterra fino al successivo riacquisto e, di conseguenza, non avrebbe potuto essere contabilizzato tra le riserve della Banca d'Italia. Con il rientro al gold standard, l'Italia annoverò l'oro di Londra tra le riserve della propria banca centrale, anche se detenuto all'estero, mentre gli inglesi ritenevano che l'oro appartenesse - seppur temporaneamente - alla Banca d'Inghilterra e, pertanto, considerarono i dati sulle riserve italiane gonfiati. Commentando in un discorso pubblico i risultati dell'accordo di ristrutturazione del debito di guerra con l'Italia, re Giorgio V affermò che esso era stato raggiunto on fair and honourable terms, ma la questione dell'oro rimase, in realtà, in sospeso ${ }^{49}$.

Per Volpi il raggiungimento degli accordi di Washington e Londra rappresentò un grande successo politico, visto che l'Italia ottenne un haircut di circa l'84\% del proprio debito di guerra ${ }^{50}$. Del resto, per Mussolini la questione aveva tutti gli aspetti di un esame di maturità internazionale attraverso cui mostrare la credibilità del regime e il ministro seppe impersonare, grazie alle sue doti, sia la posizione di tecnico esperto che di politico mediatore tra interessi diversi. Il fatto di essere un uomo d'affari, e non un accademico come De' Stefani, sicuramente aiutò Volpi, per natura e per esperienza più disponibile a individuare le soluzioni più consensuali ${ }^{51}$. L'Italia venne trattata dagli USA con condizioni particolarmente favorevoli rispetto ad altri paesi debitori. Per esempio, la Gran Bretagna beneficiò di un taglio di circa il 30\% del debito, il Belgio del 50\% e la Francia del $60 \%^{52}$. Molto probabilmente il trattamento di favore accordato all'Italia fu dovuto al fatto che a metà degli anni Venti Mussolini era ben visto oltreoceano $\mathrm{e}$ percepito come un leader in grado di assicurare stabilità politica e sociale all'Italia, una condizione indispensabile per gli investimenti americani in Europa ${ }^{53}$. Sicuramente anche l'obiettivo del ritorno al gold standard aiutò le fortune dell' Italia. Parallelamente alla riapertura dei negoziati sul debito di guerra, si svolse la trattativa per l'emissione di un prestito governativo a lungo termine da parte della casa Morgan (il prestito Kingdom of Italy), la più importante operazione finanziaria lanciata sul mercato americano nel 1925 a favore di un governo straniero $^{54}$. L'ammontare del prestito era di 100 milioni di dollari, emesso a 94,5 e a un tasso del 7 per cento ${ }^{55}$. L'operazione, il cui obiettivo era la stabilizzazione de facto

49 5,9 tonnellate furono restituite tra il 1928 e il 1931. Roselli, Money and Trade Wars in Interwar Europe, cit., p. 63.

so Astore, Fratianni, 'We Can't Pay': How Italy Dealt with War Debts after World War I, cit., p. 205.

${ }_{51}$ Segreto, Giuseppe Volpi di Misurata al Ministero delle finanze: tecnocrate o politico?, cit., p. 20.

52 G.G. Migone, Gli Stati Uniti e il fascismo. Alle origini dell'egemonia americana in Italia, Feltrinelli, Milano 1980, pp. 72-73.

53 Ibidem.

54 P.F. Asso, L'Italia e i prestiti internazionali, 1919-1931, in Ricerche per la storia della Banca d'Italia, vol. III. Finanza internazionale, vincolo esterno e cambi, 1919-1939, Laterza, Roma-Bari 1993, p. 240.

55 La somma effettivamente acquistata dal governo italiano era di 90 milioni, in quanto 4,5 milioni di dollari erano destinati a coprire i costi finanziari dell'operazione, primo fra tutti 
del tasso di cambio della lira, fu concepita inizialmente da Alberto Beneduce e fu portata a termine da Volpi come appendice alla missione italiana a Washington; il direttore della Banca d'Italia Bonaldo Stringher venne informato solo a cose fatte ${ }^{56}$. Dopo l'accordo di ristrutturazione del debito e il prestito Morgan, i capitali statunitensi cominciarono ad arrivare in Italia: da quasi zero nel 1925, il flusso di capitali arrivò a superare i 460 milioni di dollari nel $1930^{57}$.

È interessante notare come il condono da parte degli Stati Uniti venne presentato all'opinione pubblica americana, ostile a qualsiasi forma di concessione, come una ristrutturazione del debito. Rinegoziare il debito di guerra allungando di molto lo scadenzario dei pagamenti e accordare tassi di interesse relativamente bassi significava nei fatti ridurne sensibilmente il valore attuale, ma ciò non era immediato per l'elettore medio, poco familiare con le nozioni basilari di economia, che avrebbe invece concentrato la sua attenzione sul valore nominale del debito - rimasto immutato - e non sul suo valore attuale, che non a caso non venne enfatizzato dalla stampa statunitense.

Sistemati i debiti di guerra, il problema più urgente era la stabilizzazione monetaria. Mussolini riteneva inconcepibile che la piena stabilità politica, il riequilibrio della finanza pubblica, la sistemazione dei prestiti esteri non trovassero adeguata espressione nella quotazione della lira e temeva che un cambio svalutato offrisse argomenti agli oppositori ${ }^{58}$. La severa stretta deflazionistica che accompagnò la fissazione della lira a 'quota 90' aveva provocato, come è ovvio, una scarsità di circolante e molti istituti bancari, imprese e industriali che in precedenza avevano impiegato in Bot le proprie disponibilità liquide, richiedevano il rimborso dei buoni in scadenza, anziché il rinnovo ${ }^{59}$. Il governo optò per una conversione forzosa di una parte notevole del debito pubblico (il cosiddetto 'prestito del Littorio'). L'operazione di consolidamento prevedeva la conversione obbligatoria dei buoni quinquennali e settennali e la conversione volontaria dei buoni novennali in circolazione in un consolidato $5 \%$ non redimibile. Nel giro di un anno tutto il debito fluttuante venne eliminato, ma la conversione forzosa rappresentò una sorta di default parziale e fece perdere credibilità alle istituzioni finanziarie italiane ${ }^{60}$. In seguito alla conversione forzosa, il governo sperimentò sempre più difficoltà nel finanziamento a breve termine.

Nel corso degli anni successivi al raggiungimento degli accordi di Washington e di Londra i versamenti dell'Italia per i debiti di guerra procedettero rego-

la commissione, abbastanza esosa, di Morgan. Ibidem.

56 M. Franzinelli, M. Magnani, Beneduce. Il finanziere di Mussolini, Mondadori, Milano 2009, p. 163.

57 D.F. Schmitz, The United States and Fascist Italy, University of North Carolina Press, Chapel Hill and London 1988, pp. 96, 109.

58 Cotula, Spaventa (a cura di), La politica monetaria tra le due guerre, 1919-1935, cit., p. 140.

59 Ministero del tesoro, Direzione generale del debito pubblico, Relazione del direttore generale alla commissione parlamentare di vigilanza. Il debito pubblico in Italia, 1861-1987, vol. I, cit., p. 38.

60 A. Alesina, The End of Large 'Public Debts, in F. Giavazzi, L. Spaventa (eds.), High Public Debt: The Italian Experience, Cambridge University Press, Cambridge 1988. 
larmente (si veda Tab. 2). Per la loro corretta gestione venne addirittura creata una cassa ad hoc presso la Cassa depositi e prestiti ${ }^{61}$. Il funzionamento della cassa - tecnicamente chiamata 'Cassa di ammortamento dei debiti di guerra' - era molto semplice e si basava sul principio che le riparazioni tedesche ricevute dall'Italia servissero al pagamento del debito estero nei confronti degli Stati Uniti e dell'Inghilterra (si veda Tab. 3). Di lì a poco, però, la Grande depressione segnò un grande spartiacque nella vicenda di debiti di guerra e riparazioni. Più in generale, gli effetti della crisi si fecero pesantemente sentire sul bilancio italiano a partire dal 1932, ed infine le avventure belliche di Mussolini e la Seconda guerra mondiale provocarono disavanzi di bilancio crescenti fino al collasso finale ${ }^{62}$.

Tabella 2 - Somme pagate agli Stati Uniti e alla Gran Bretagna per i debiti di guerra. [Fonte: Astore, Fratianni, 'We Can't Pay': How Italy Dealt with War Debts after World War I, cit., p. 219]

\begin{tabular}{|c|c|c|c|c|}
\hline Anno & Descrizione & $\begin{array}{l}\text { Ammontare } \\
\text { in valuta estera }\end{array}$ & $\begin{array}{l}\text { Tasso di cambio } \\
\text { medio mensile }\end{array}$ & $\begin{array}{l}\text { Controvalore } \\
\text { in lire }\end{array}$ \\
\hline 1925 & Debito statunitense (dollari) & 199.466 & & 4.986 .658 \\
\hline 1926 & Debito statunitense (dollari) & 5.000 .000 & & 125.000 .000 \\
\hline 1926 & Debito inglese (sterline) & 4.000 .000 & & 523.120 .000 \\
\hline 1927 & Debito statunitense (dollari) & 5.000 .000 & & 111.600 .000 \\
\hline 1927 & Debito inglese (sterline) & 4.000 .000 & & 400.274 .000 \\
\hline 1928 & Debito statunitense (dollari) & 5.000 .000 & & 95.000 .000 \\
\hline 1928 & Debito inglese (sterline) & 4.125 .000 & & 381.897 .000 \\
\hline 1929 & Debito statunitense (dollari) & 5.000 .000 & & 95.000 .000 \\
\hline 1929 & Debito inglese (sterline) & 4.250 .000 & & 393.998 .000 \\
\hline 1930 & Debito statunitense (dollari) & 6.260 .625 & & 118.951 .875 \\
\hline 1930 & Debito inglese (sterline) & 5.312 .500 & & 491.246 .875 \\
\hline 1931 & Debito statunitense (dollari) & 13.360 .625 & & 253.851 .875 \\
\hline 1931 & Debito inglese (sterline) & 2.125 .000 & & 196.498 .750 \\
\hline $\begin{array}{l}\text { dicem- } \\
\text { bre } 1932\end{array}$ & $\begin{array}{l}\text { Debito statunitense (dollari) - } \\
\text { pagamento parziale (c.d.token) }\end{array}$ & 1.200 .000 & 19,55 & 23.460 .000 \\
\hline $\begin{array}{l}\text { giugno } \\
1933\end{array}$ & $\begin{array}{l}\text { Debito statunitense (dollari) - } \\
\text { pagamento parziale (c.d.token) }\end{array}$ & 1.000 .000 & 15,71 & 15.710 .000 \\
\hline $\begin{array}{l}\text { dicembre } \\
1933\end{array}$ & $\begin{array}{l}\text { Debito statunitense (dollari) - } \\
\text { pagamento parziale (c.d.token) }\end{array}$ & 1.000 .000 & 12,15 & 12.150 .000 \\
\hline
\end{tabular}

${ }_{61}$ Regio Decreto Legge 3 marzo 1926, n. 332.

62 Ministero del tesoro, Direzione generale del debito pubblico, Relazione del direttore generale alla commissione parlamentare di vigilanza. Il debito pubblico in Italia, 1861-1987, vol. I, cit., p. 38. 
Tabella 3 - Riparazioni di guerra ricevute dall'Italia, 1919-1931. [Fonte: Astore, Fratianni, 'We Can't Pay': How Italy Dealt with War Debts after World War I, cit., p. 219]

\begin{tabular}{lllll}
\hline Anni & Descrizione & Marchi oro & $\begin{array}{l}\text { Tasso di } \\
\text { cambio medio }\end{array}$ & $\begin{array}{l}\text { Riparazioni ricevute } \\
\text { (in lire) }\end{array}$ \\
\hline $1919-1923$ & $\begin{array}{l}\text { Riparazioni prima del } \\
\text { piano Dawes }\end{array}$ & 428.652 .459 & 0,1805 & 77.357 .838 \\
\hline $1924-1929$ & $\begin{array}{l}\text { Riparazioni secondo il } \\
\text { piano Dawes }\end{array}$ & 555.130 .021 & 5,2353 & 2.906 .290 .703 \\
\hline $1929-1930$ & $\begin{array}{l}\text { Riparazioni nel periodo } \\
\text { di transizione }\end{array}$ & 83.513 .207 & 4,553 & 380.235 .631 \\
\hline $1930-1931$ & $\begin{array}{l}\text { Riparazioni secondo il } \\
\text { piano Young }\end{array}$ & 228.046 .600 & 4,5485 & 1.037 .269 .960 \\
\hline Totale & & 1.295 .341 .678 & & 4.401 .154 .132 \\
\hline
\end{tabular}

\section{La conferenza d Losanna e i debiti di guerra italiani}

Nel giugno 1931 il presidente degli Stati Uniti Herbert Hoover, preoccupato dalla situazione generale europea e in particolare da quella della Germania e dell'Austria, propose la sospensione di un anno per tutti i debiti derivanti dalla guerra. Tale proposta, che passò alla storia come 'moratoria Hoover', venne inizialmente accettata senza troppe criticità dall'opinione pubblica statunitense perché si riteneva avrebbe dato una spinta al commercio americano all'indomani della crisi ${ }^{63}$. Tuttavia, con l'aggravarsi della Depressione, il clima politico si fece molto più teso e anche se, nel dicembre 1931, la moratoria venne ufficialmente ratificata dal Congresso, venne emanata simultaneamente una risoluzione in cui si esprimeva la contrarietà statunitense alla cancellazione dei debiti di guerra. Un anno dopo la moratoria Hoover venne convocata - su invito formale del governo inglese - una conferenza al fine di raggiungere un accordo definitivo sulle riparazioni tedesche; la conferenza si tenne a Losanna dal 16 giugno al 9 luglio 1932. La delegazione italiana, che comprendeva il Ministro degli esteri Dino Grandi, Alberto Pirelli e Alberto Beneduce, sostenne in sede diplomatica la posizione di legare a doppio filo le riparazioni ai debiti di guerra ${ }^{64}$. In particolare, venivano evidenziate l'insufficienza di una moratoria, anche se prolungata, e la necessità di un colpo di spugna nei confronti delle riparazioni tedesche, ma si subordinava tale colpo di spugna alla cancellazione dei debiti di guerra da parte della Gran Bretagna e degli Stati Uniti ${ }^{65}$. In sostanza, la strategia diploma-

63 W. Lippmann, The United States in World Affairs: An Account of American Foreign Relations, Harper and Brothers, New York and London 1935, p. 5.

64 De Cecco, L'Italia e il sistema finanziario internazionale, 1919-1936, cit., p. 643.

65 Documenti diplomatici italiani (d'ora in avanti DDI), Settima serie, vol. XII, n.116, Il Ministro degli esteri, Grandi, e il Ministro delle finanze, Mosconi, al capo del governo Mussolini, 24 giugno 1932, pp. 156-158. 
tica dell'Italia prevedeva che gli Stati europei si rimettessero reciprocamente i propri debiti e condonassero le riparazioni alla Germania, per poi presentarsi coesi nei confronti degli Stati Uniti, ai quali avrebbero chiesto di fare altrettanto. È questo un punto sul quale la delegazione italiana insiste particolarmente:

Il regolamento definitivo delle riparazioni importa come conseguenza naturale quello dei debiti di guerra. [...] Il Governo italiano ha sempre ritenuto che l'interdipendenza tra il problema dei debiti di guerra e quello delle riparazioni sia un aspetto essenziale del problema. Le riparazioni infatti costituiscono per molti paesi un'indispensabile contropartita finanziaria per i pagamenti dovuti a rimborso dei debiti di guerra ${ }^{66}$.

La conferenza si chiuse con un accordo con la Germania in base al quale quest'ultima si impegnava a consegnare alla Banca dei regolamenti internazionali obbligazioni tedesche pari a 3 miliardi di marchi oro, poco meno del $3 \%$ della somma totale dei pagamenti previsti dal piano Young ${ }^{67}$. Tradizionalmente la letteratura sui debiti di guerra dell'Italia ha enfatizzato i risultati raggiunti a Losanna, considerando la conferenza l'atto finale dell'annosa questione dei debiti e delle riparazioni ${ }^{68}$; ad esempio, nella ricostruzione della serie storica del debito pubblico italiano di Francese e Pace, che rappresenta il benchmark della letteratura quantitativa in materia, i debiti di guerra italiani vengono azzerati nel 1932, a seguito della conferenza ${ }^{69}$. In realtà quest'ultima rappresentò più che altro un tentativo da parte dei paesi debitori di chiedere la cancellazione dei debiti e lasciò di fatto la situazione in uno stato di limbo ${ }^{70}$. Laccordo di Losanna infatti non venne mai ratificato, e Gli Stati Uniti, che non erano formalmente coinvolti nella gestione delle riparazioni tedesche, non parteciparono nemme-

66 Lettera di Beneduce al presidente della Conferenza di Losanna James Ramsay MacDonald, Losanna 25 giugno 1932, riportato in De Cecco, L'Italia e il sistema finanziario internazionale, 1919-1936, cit., pp. 642-646.

67 Il piano Young, preparato nel 1929 e approvato formalmente il 30 gennaio 1930, sostituì il piano Dawes e ridusse il valore delle riparazioni tedesche a 110 miliardi di Reichsmark, a cui si aggiungevano 5,2 miliardi di conferimenti in natura. L'intero importo doveva essere pagato in 59 anni con delle annualità inferiori al piano Dawes. Quest'ultimo era entrato in vigore nel 1924 e prevedeva il pagamento annuale di un miliardo di marchi-oro sino al 1928 e di 2,5 in seguito. Il piano era sostenuto da un prestito internazionale in dollari - il prestito Dawes - pari a 800 milioni di Reichsmark, emesso con il coordinamento della J.P. Morgan \& Co., che permise la stabilizzazione della divisa tedesca e il ristabilimento della fiducia nell'economia del paese. Cfr. Roselli, Money and Trade Wars in Interwar Europe, cit., pp. 39-42 e Y. Cassis, Le capitali della finanza. Uomini e città protagonisti della storia economica, Francesco Brioschi Editore, Milano 2008, pp. 165-166.

68 Una significativa eccezione è rappresentata dallo studio di C.M. Reinhart e C. Trebesch, $A$ Distant Mirror of Debt, Default, and Relief, «Munich Discussion Paper», 49, 2014.

${ }_{69}$ M. Francese, A. Pace, Il debito pubblico italiano dall'Unità a oggi. Una ricostruzione della serie storica, «Questioni di economia e finanza», Banca d'Italia, 31, 2008, p. 17.

70 G. Toniolo, Central Bank Cooperation at the Bank for International Settlements, 1930-1973, con la collaborazione di P. Clement, Cambridge University Press, New York 2005, p. 131. 
no alla conferenza ${ }^{71}$. Particolarmente eloquente a questo proposito è una lettera congiunta del Ministro degli esteri Grandi e del Ministro delle finanze Mosconi a Mussolini, scritta proprio mentre la conferenza era in corso di svolgimento:

La politica dell'America in materia di debiti di guerra, politica certo influenzata dalla situazione elettorale, ma forse anche dall'idea di tenere in pugno la questione dei debiti come arma di negoziazione e di imposizione verso i singoli Stati europei in materia di disarmo o per altre negoziazioni, resta uno dei fattori dominanti del negoziato. Taluni ritengono che, se l'Europa desse senz'altro il buon esempio, l'America sarebbe trascinata a seguirla; altri ritengono invece che non si possa correre questo rischio, anche perché il Congresso americano è una bestia pericolosa [...] si dice che, d'altronde, l'America ha fatto sapere che una cancellazione completa delle riparazioni nel momento attuale sarebbe dannosa per ottenere la cancellazione dei debiti sia perché siamo in periodo elettorale e sia perché darebbe l'impressione all'opinione pubblica americana che l'Europa ha scaricato sul contribuente americano tutto il sacrificio ${ }^{72}$.

Gli esiti di Losanna erano, dunque, ben lontani da quanto auspicato dall'Italia e rappresentavano in qualche modo un fallimento politico. Il disappunto venne anche reso pubblico dalla delegazione italiana, che così si espresse ufficialmente:

La cancellazione delle riparazioni tedesche toglierà all'Italia un margine netto di non poca entità, sul quale il bilancio dello Stato aveva contato come parziale compenso alle non eque ripartizioni del passato. Questo saldo non rappresenta tuttavia la misura del sacrificio che importerà all'Italia la cancellazione delle riparazioni germaniche. L'opinione pubblica italiana ha la profonda convinzione del trattamento sfavorevole fatto in tutti i campi all'Italia nella ripartizione delle riparazioni di guerra ${ }^{73}$.

\section{Debiti nel dimenticatoio? Oltre la Grande depressione}

La definitiva sistemazione dei debiti di guerra non solo non venne risolta nel corso della Conferenza di Losanna, ma nemmeno negli anni successivi quando la questione dei pagamenti si ripropose a più riprese. Nel novembre 1932 gli

71 Parallelamente all'accordo principale venne siglato un accordo parallelo tra i rappresentati di Belgio, Francia, Italia e Gran Bretagna, che subordinò la ratifica dell'accordo principale al raggiungimento di una soluzione soddisfacente sui debiti di guerra. L'accordo parallelo, siglato dal ministro Mosconi per l'Italia, venne interpretato negli Stati Uniti come un tentativo di creare un fronte comune fatto dai paesi europei per non pagare i propri debiti. Per i testi originali degli accordi di Losanana, cfr. Carnegie Endowment for International Peace, The Lausanne Agreement. The Text of the Final Act of the Lausanne Conference, July 9, 1932, and Texts of Further Documents Related to the Settlement Reached at Lausanne, June 16-July 9, 1932, New York 1932, pp. 334-350.

72 DDI, Settima serie, vol. XII, n.116, Il Ministro degli esteri, Grandi, e il Ministro delle finanze, Mosconi, al capo del governo Mussolini, 24 giugno 1932, pp. 156-158.

73 Lettera di Beneduce al presidente della Conferenza di Losanna James Ramsay MacDonald, Losanna 25 giugno 1932, cit. 
inglesi chiesero agli Stati Uniti la sospensione della rata dovuta a dicembre. La richiesta inglese fu seguita da altri paesi, come la Francia e il Belgio, ma gli Stati Uniti rifiutarono ${ }^{74}$. La Gran Bretagna decise così di corrispondere un pagamento parziale (cosiddetto token payments), la stessa cosa fece l'Italia, mentre la Francia optò per il default un giorno prima della scadenza del 15 dicembre ${ }^{75}$. Nel giugno 1933 la Gran Bretagna e l'Italia optarono ancora una volta per un pagamento simbolico e la stessa cosa si ripeté a dicembre ${ }^{76}$. Furono le ultime rate corrisposte. Nella difficile temperie della Grande crisi degli anni Trenta, tra la fine del 1931 e il 1934 numerose misure furono adottate per restringere la libertà degli scambi commerciali con l'estero. Sotto questo profilo, sintomatico fu il fallimento della conferenza economica di Londra del 1933, con la quale si era cercato di dare una soluzione a problemi comuni: l'urgenza di stabilizzare i cambi e di ridurre le tariffe protezionistiche. Nel corso della conferenza, tenutasi a giugno, Roosevelt rifiutò di stabilizzare il dollaro, che era uscito dal gold standard in aprile ${ }^{77}$. A prevalere furono gli interessi nazionali e la conferenza segnò il fallimento di ogni tentativo di restaurare regole condivise a livello internazionale, oltre che la fine del gold standard (sebbene allora non se ne avesse piena consapevolezza). La sterlina, tornata alla convertibilità aurea nel 1925, era già svalutata nel 1931. Francia e Italia, insieme a Belgio, Svizzera, Olanda e Polonia, costituirono il blocco dell'oro, preferendo rimanere ancorate alla base aurea, ma furono costrette a svalutare nel 1936. La Germania mantenne il tasso di cambio, ma tramite strettissimi controlli valutari e rendendolo quasi fittizio. L'inasprimento dei dazi protettivi contro le importazioni si accompagnò a restrizioni valutarie in diversi paesi $^{78}$. Nel dicembre 1934 in Italia venne introdotto il monopolio sui cambi, mentre nel 1935 vennero contingentate le importazioni. Nello stesso anno venne costituita presso la presidenza del Consiglio, sotto il diretto comando del duce, la Sovrintendenza allo scambio delle valute. I provvedimenti furono dettati soprattutto dall'esigenza di cercare di porre un

74 B. Eichengreen, Golden Fetters: The Gold Standard and the Great Depression, 1919-1939, Oxford University Press, Oxford 1992, p. 319.

75 La Camera dei deputati francese ripudiò l'accordo di ristrutturazione del debito ratificato nel 1929. La decisione venne comunicata formalmente da una lettera dell'ambasciatore francese De Laboulaye al segretario di Stato americano. Il testo originale della lettera è riportato in Astore, Fratianni, 'We Can't Pay': How Italy Dealt with War Debts after World War I, cit., pp. 217-218.

76 Reinhart and Trebesch, A Distant Mirror of Debt, Default, and Relief, cit., p. 24.

77 Il segretario di Stato statunitense Cordell Hull così si espresse nel corso della conferenza: «The sound internal economic system of a nation is a greater factor in its well-being than the price of its currency. [...] Restoration of world trade is important, [but] temporary exchange fixing is not the true answer. We must rather mitigate existing embargoes to make easier the exchange of products which one nation has and other nations have not». Il rifiuto statunitense suscitò una reazione molto negativa da parte di Alberto Beneduce. Cfr. Roselli, Money and Trade Wars in Interwar Europe, cit., p. 98.

78 B. Eichengreen, J. Sachs, Exchange Rates and Economic Recovery in the 1930s, «Journal of Economic History», 45 (4), 1985, pp. 925-946, tabella 1. 
freno al depauperamento delle riserve della Banca d'Italia, già iniziato a partire dal $1928^{79}$. Con i controlli sui cambi adottati da molti paesi e la contrazione dei redditi causata dalla depressione, che ridussero entrambi il flusso di valuta, l'Italia entrò in default nel 1934. Sul fronte del debito pubblico interno, la lenta crescita dopo la grande crisi incominciata nel 1929 e le pressioni deflazionistiche portarono a un aumento dell'onere del debito pubblico a lungo termine. Di conseguenza, nei primi anni Trenta, il fascismo portò avanti la politica delle conversioni forzose, nonostante fosse molto negativa in termini di reputazione. Con l'operazione di conversione del 3 febbraio 1934 si offrì un titolo redimibile al 3,50\%, ma il diritto di scelta tra conversione e rimborso non si poté di fatto esercitare, così che si trattò di una conversione solo apparentemente volontaria ${ }^{80}$.

Nel frattempo l'atteggiamento degli Stati Uniti era diventato sempre più isolazionista al punto che, nell'aprile del 1934, venne emanato il Debt Default Act - anche conosciuto come Johnson Act dal nome del suo proponente, il senatore Hiram Johnson -, che proibiva ulteriori prestiti a quegli Stati che fossero insolventi nel pagamento dei debiti di guerra (anche pagamenti simbolici erano ritenuti insufficienti) ${ }^{81}$. In seguito all'emanazione del Johnson Act, la Gran Bretagna, con una nota del 4 giugno 1934, comunicò l'intenzione di sospendere i pagamenti fino a quando la questione non fosse posta nuovamente sul tavolo delle trattative ${ }^{82}$. Nelle parole del primo ministro inglese Ramsay MacDonald, continuare a pagare sarebbe stata una follia ${ }^{83}$. L'Italia di lì a poco decise di fare lo stesso ${ }^{84}$; la notizia venne comunicata anche alla stampa, ma - chiaramente non venne enfatizzata. Non furono casi isolati: nel 1934 fecero default tutti i debitori europei, ad eccezione della Finlandia ${ }^{85}$. Quasi simultaneamente il Reich tedesco annunciò che dopo il 30 giugno 1934 non avrebbe più continuato con il pagamento delle riparazioni ${ }^{86}$.

79 Banca d'Italia, Adunanza generale ordinaria degli azionisti tenuta in Roma il giorno 30 marzo 1935-XIII, Tipografia della Banca d'Italia, Roma 1935, p. 12.

80 Marinkov, Conquering the debt mountain. Financial repression and Italian debt in the interwar period, cit., p. 191; Ministero del tesoro, Direzione generale del debito pubblico, Relazione del direttore generale alla commissione parlamentare di vigilanza. Il debito pubblico in Italia, 1861-1987, vol. I, cit., p. 41-42.

81 M. Folly, N. Palmer, The A to Z of U.S. Diplomacy from World War I through World War II, Scarecrow Press, Metuchen, NJ 2010, p. 84. Il provvedimento legislativo rifletteva l'orientamento dell'opinione pubblica statunitense nei confronti dei debitori europei, nonostante l'establishment e, in particolare, il presidente Roosevelt avessero un atteggiamento molto meno intransigente. Cfr. Documents on British Foreign Policy (d'ora in avanti DBFP), seconda serie, vol. V, n. 598, pp. 853-855.

82 DBFP, seconda serie, vol. VI, n. 594, p. 935.

83 R. Self, Perception and Posture in Anglo-American Relations: The War Debt Controversy in the 'Official Mind', 1919-1940, «International History Review», 29 (2), 2007, p. 286.

84 Il testo originale della lettera si trova in DDI, Settima Serie, vol. XV, n. 397, pp. 419-420.

85 Reinhart, Trebesch, A Distant Mirror of Debt, Default, and Relief, cit., tab. 1, p. 20.

86 Toniolo, Central Bank Cooperation at the Bank for International Settlements, 1930-1973, cit., p. 154. 
Il pagamento dei debiti di guerra venne riproposto sporadicamente negli anni successivi, ma con scarsa convinzione da parte di tutti i paesi coinvolti. Ad esempio, a metà del $1936 \mathrm{al}$ Congresso vennero formulate diverse proposte che avrebbero dovuto facilitare il pagamento alle ex potenze alleate, ma esse non trovarono seguito (alcune di queste erano del resto molto inverosimili) ${ }^{87}$. L'episodio è tuttavia sintomatico del fatto che gli Stati Uniti non fossero disposti a dimenticare i debiti, sebbene la questione - pur sussistendo ancora dal punto di vista giuridico - rimanesse sostanzialmente irrisolta ${ }^{88}$.

La sistemazione del debito di guerra inglese seguì un percorso in parte differente. La Gran Bretagna si trovava infatti nella duplice posizione di essere debitore nei confronti degli Stati Uniti e creditore nei confronti degli altri Alleati. A Losanna inizialmente si registrò una certa diffidenza nei confronti dell'atteggiamento diplomatico degli inglesi e in particolare circa la loro volontà di riconoscere, o meno, il legame tra debiti e riparazioni:

Quello che è essenziale per noi è che nell' 'istesso momento in cui noi rimettiamo i nostri crediti alla Germania, la Gran Bretagna deve rimettere i suoi crediti a noi, e siano parimenti adottate delle comuni misure di salvaguardia nei riguardi dell'America. La Gran Bretagna ci vuole portare invece tutti quanti a liberare la Germania, e mantenere intatti nei riguardi dei suoi creditori europei i suoi diritti, in attesa di conoscere quello che farà l'America nei riguardi di tutti. Ma questo non è il 'colpo di spugna' o meglio è un colpo di spugna unilaterale, a solo vantaggio della Germania e della Gran Bretagna. Non credo che noi possiamo accettarlo $^{89}$.

Nel corso della conferenza, tuttavia, la Gran Bretagna, pur non accordando esplicitamente un condono ai propri debitori, di fatto raggiunse con questi ultimi un accordo tacito, in base al quale non avrebbe più richiesto i pagamenti e avrebbe concentrato i suoi sforzi diplomatici sulla cancellazione del debito statunitense. Diversi documenti supportano questa interpretazione. Soltanto qualche giorno dopo rispetto alla lettera sopracitata, secondo quanto riferito dal Ministro degli esteri Grandi, nel corso di un colloquio privato MacDonald avrebbe dato la sua parola d'onore che l'Inghilterra era disponibile a cancellare i suoi debiti nei confronti dell'Italia, ma per motivi eminentemente politici non

87 «Corriere della Sera», 16 giugno 1936.

88 Nel luglio 1937 l'ambasciatore a Washington Suvich, riferendo al Ministro degli esteri Ciano un suo colloquio con il segretario di Stato Hull, scrisse: [Sulla regolazione dei debiti di guerra Hull] $\mathrm{mi}$ ha detto: «Se io andassi nelle varie capitali di Europa, Londra, Parigi e Roma, a trattare per una regolazione dei debiti e regolassi la questione, ad esempio sulla base di un taglio del 50, del 20 o del 10 per cento, ritornando al mio Paese io sarei considerato un traditore degli interessi dei taxpayers americani. Viceversa, se alla stessa soluzione si arrivasse per un accordo di carattere generale in cui ogni Paese portasse il proprio contributo, la cosa non solleverebbe obiezioni». DDI, ottava serie, vol. VII, n. 39, pp. 44-45.

89 Il Ministro degli esteri, Grandi, e il Ministro delle finanze, Mosconi, al capo del governo, Mussolini, 24 giugno 1932, in DDI, settima serie, vol. X, n. 116, pp.156-158. 
poteva dichiararlo esplicitamente nell'accordo di Losanna ${ }^{90}$. La consapevolezza (quasi la certezza) italiana che non sarebbero stati richiesti ulteriori pagamenti da parte inglese appare completamente maturata agli inizi del $1933^{91}$. Talvolta in sede diplomatica la mancata formalizzazione della rinuncia ai propri crediti veniva utilizzata come strumento contrattuale da parte della Gran Bretagna, ma a quanto pare con scarsa efficacia ${ }^{92}$. L'Italia dunque non onorò più i propri debiti nei confronti della Gran Bretagna dopo la conferenza di Losanna, perché questi non furono più richiesti. Quel che si creò fu un interstizio tra il piano legale/ formale - il solo comunicabile all'opinione pubblica - e quello sostanziale, che si sviluppava a livello di trattative diplomatiche: di fatto ci fu un default silente.

Se l'Italia non pagò mai più i propri debiti di guerra, non va dimenticato che fra il 1925 e il 1933 vennero emessi sui mercati interazionali, da enti e società italiane, prestiti per circa 370 milioni di dollari, che erano stati collocati quasi esclusivamente sul mercato statunitense ${ }^{93}$. Nel gennaio 1947, il Crediop e l'Icipu ricevettero l'incarico dal governo italiano di negoziare il piano di ristrutturazione del debito estero con gli Stati Uniti. Complessivamente il piano di sistemazione riguardava 17 emissioni di prestiti prebellici, fra le quali erano compresi i prestiti emessi da Icipu e Crediop nel 1926 e nel 1927. Per l'economia italiana si trattava di un'operazione di importanza strategica, in quanto era uno dei presupposti per normalizzare i rapporti fra governo e imprese italiane da un lato, $\mathrm{e}$ mercato finanziario americano dall'altro, in vista dell'approvazione e dell'inaugurazione del piano Marshall. La sistemazione dei debiti prebellici comportò la conversione del vecchio prestito Morgan Kingdom of Italy in un nuovo titolo Republic of Italy, per un ammontare complessivo di 39,6 milioni di dollari. Inoltre i prestiti emessi a favore di alcune municipalità o di enti pubblici garantiti dallo Stato (Consorzio di credito per le opere pubbliche, città di Roma, città di Milano, Istituto di credito fondiario delle Venezie) vennero convertiti in una nuo-

90 Il Ministro degli esteri, Grandi, al capo del Governo, Mussolini, Losanna, 3 luglio 1932, in DDI, settima serie, vol. XII, n. 139, pp. 190-191. L'impegno inglese appare confermato anche dal capo di gabinetto del Ministero degli esteri Aloisi a Mussolini (DDI, settima serie, vol. XII, n. 524, pp. 634-635).

91 L'ambasciatore a Londra, Grandi, al Capo del governo e Ministro degli esteri, Mussolini, Londra, 3 gennaio 1933 (DDI, settima serie, vol. XIII, n. 3, pp. 3-5).

92 Nel febbraio 1933 Grandi, nella sua nuova veste di ambasciatore a Londra, scrive a Mussolini: «Vediamo punto per punto in quale fase si trovi lo svolgimento della politica estera inglese [...] Ogni tanto, a scopo polemico, MacDonald o Sir John Simon, o Neville Chamberlain, presentano l'ipotesi che ove non si giungesse a un regolamento del debito con l'America, bisognerebbe rivedere gli accordi di Losanna, ma né essi né alcuno nel paese crede in tale possibilità. È questa semplicemente una delle forme nelle quali essi esprimono il concetto fondamentale in base al quale essi vorrebbero che gli Stati Uniti misurassero la capacità di pagamento dell'Inghilterra: gli accordi di Losanna non si possono toccare» (DDI, settima serie, vol. XIII, n. 144, L'ambasciatore a Londra, Grandi, al Capo del governo e Ministro degli esteri, Mussolini, Londra, 27 febbraio 1933, p. 145).

93 P.F. Asso, M. De Cecco, Storia del Crediop: tra credito speciale e finanza pubblica, 1920-1960, Laterza, Roma-Bari 1994, p. 337. 
va serie di obbligazioni in dollari emesse dal Crediop alle stesse condizioni del Republic of Italy, per un totale di 37,2 milioni, che furono offerte in cambio dei titoli non rimpatriati e degli interessi non corrisposti. Nella sostanza la sistemazione prevedeva la rinuncia da parte del creditore ad alcune condizioni originarie, configurandosi come una debt forgiveness ${ }^{94}$. Si trattava di un tassello dei più ampi accordi 'Lombardo-Lovett' stipulati tra Italia e Stati Uniti nell'agosto del 1947 e che seguirono la missione diplomatica oltreoceano del primo ministro Alcide De Gasperi, un punto di svolta nei rapporti diplomatici italo-statunitensi del secondo dopoguerra. Eravamo già nel contesto del piano Marshall, una pagina nettamente diversa da quella degli anni tra le due guerre mondiali, che aveva invece visto il fallimento della collaborazione economica internazionale.

94 Nello specifico: 1) rinuncia alla capitalizzazione dell'interesse non onorato negli anni del 'default' e annullamento di tutti gli interessi di mora; 2) allungamento della scadenza e avvio del nuovo piano di ammortamento solamente a partire dal quinto anno; 3) la riduzione del tasso di interesse effettivo. Ivi, p. 359. 